\title{
q-Bernstein-Type Polynomials for Functions of Two Variables with Their Generating and Interpolation Functions
}

\author{
Mehmet ACIKGOZ ${ }^{1}$, Erdoğan ŞEN ${ }^{2}$, Serkan ARACI ${ }^{3, *}$ \\ ${ }^{1}$ University of Gaziantep, Faculty of Arts and Science, Department of Mathematics, Gaziantep, Turkey \\ ${ }^{2}$ Department of Mathematics, Faculty of Science and Letters, Namik Kemal University, Tekirdağ, Turkey \\ ${ }^{3}$ Atatürk Street, Hatay, Turkey \\ *Corresponding author: mtsrkn@hotmail.com
}

Received September 15, 2013; Revised October 25, 2013; Accepted November 04, 2013

\begin{abstract}
The aim of this paper is to give a new approach to modified $q$-Bernstein polynomials for functions of two variables. By using these type polynomials, we derive recurrence formulas and some new interesting identities related to the second kind Stirling numbers and generalized Bernoulli polynomials. Moreover, we give the generating function and interpolation function of these modified $q$-Bernstein polynomials of two variables and also give the derivatives of these polynomials and their generating function.
\end{abstract}

Keywords: generating function, Bernstein polynomial of two variables, Bernstein operator of two variables, shift difference operator, q-difference operator, second kind Stirling numbers, generalized Bernoulli polynomials, Mellin transformation, interpolation function

Cite This Article: Mehmet ACIKGOZ, Erdoğan ŞEN, and Serkan ARACI, "q-Bernstein-Type Polynomials for Functions of Two Variables with Their Generating and Interpolation Functions." Turkish Journal of Analysis and Number Theory 1, no. 1 (2013): 36-42. doi: 10.12691/tjant-1-1-8.

\section{Introduction, Definitions and Notations}

In approximation theory, the Bernstein polynomials, named after their creater S. N. Bernstein in 1912, have been studied by many researchers for a long time. But nothing about generating function of Bernstein polynomials were known in the literature. Recently, Simsek and Acikgoz, ([18]), constructed a new generating function of $(q-)$ Bernstein type polynomials based on the $q$-analysis. They gave some new relations related to $(q-)$ Bernstein type polynomials, Hermite polynomials, Bernoulli polynomials of higher order and the second kind Stirling numbers. By applying Mellin transformation to this generating function they defined the interpolation function of $(q-)$ Bernstein type polynomials. They gave some relations and identities on these polynomials. They constructed the generating function for classical Bernstein polynomials, and for Bernstein polynomials for functions of two variables and gave their properties (see $[8,9,10]$, for details). In [1-7], T. Kim also gave a novel definition of $q$ Bernstein polynomials and derived not only new but also interesting properties of $q$-Bernstein polynomials. Actually, we are motivated to write this paper from Kim's arithemtic works.

Throughout this paper, we use some notations like N, $\mathrm{N}_{0}$ and $D$, where $\mathrm{N}$ denotes the set of natural numbers, $\mathrm{N}_{0}:=\mathrm{N} \bigcup\{0\}$ and $D=[0,1]$.
Let $C(D \times D)$ denotes the set of continuous functions on $D$. For $f \in C(D \times D)$

$$
\begin{aligned}
& \mathbf{B}_{n, m}(f ; x, y) \\
& :=\sum_{k=0}^{n} \sum_{j=0}^{m} f\left(\frac{k}{n}, \frac{j}{m}\right)\left(\begin{array}{l}
n \\
k
\end{array}\right)\left(\begin{array}{c}
m \\
j
\end{array}\right) x^{k} y^{j}(1-x)^{n-k}(1-y)^{m-j} \\
& =\sum_{k=0}^{n} \sum_{j=0}^{m} f\left(\frac{k}{n}, \frac{j}{m}\right) B_{k, j ; n, m}(x, y)
\end{aligned}
$$

where $\left(\begin{array}{l}n \\ k\end{array}\right)=\frac{n(n-1) \cdots(n-k+1)}{k !}$. Here $\mathbf{B}_{n, m}(f ; x, y)$ is called the Bernstein operator of two variables of order $n+m$ for $f$. For $k, j, n, m \in \mathrm{N}_{0}$, the Bernstein polynomial of two variables of degree $n+m$ is defined by

$$
\begin{aligned}
& B_{k, j ; n, m}(x, y) \\
= & \left(\begin{array}{l}
n \\
k
\end{array}\right)\left(\begin{array}{c}
m \\
j
\end{array}\right) x^{k} y^{j}(1-x)^{n-k}(1-y)^{m-j},
\end{aligned}
$$

where $x \in D$ and $y \in D$. Thus, throughout this work, we will assume that $x \in D$ and $y \in D$. Then, we easily see the following

$$
B_{k, j ; n, m}(x, y)=B_{k, n}(x, y) B_{j, m}(x, y)
$$


and they form a partition of unity; that is;

$$
\sum_{k=0}^{n} \sum_{j=0}^{m} B_{k, j ; n, m}(x, y)=1
$$

and by using the definition of Bernstein polynomials for functions of two variables, it is not difficult to prove the property given above as

$$
\sum_{k=0}^{n} \sum_{j=0}^{m} B_{k, n}(x, y) B_{j, m}(x, y)=1 .
$$

Some Bernstein polynomials of two variables are given below:

$$
\begin{aligned}
& B_{0,0 ; 1,0}(x, y)=(1-x), \\
& B_{0,0 ; 0,1}(x, y)=(1-y), \\
& B_{0,0 ; 1,1}(x, y)=(1-x)(1-y), \\
& B_{0,1 ; 1,1}(x, y)=y(1-x), \\
& B_{1,0 ; 1,1}(x, y)=x(1-y), \\
& B_{1,1 ; 1,1}(x, y)=x y .
\end{aligned}
$$

Also, $B_{k, j ; n, m}(x, y)=0$ for $k>n$ or $j>m$, because $\left(\begin{array}{l}n \\ k\end{array}\right)=0$ or $\left(\begin{array}{l}m \\ j\end{array}\right)=0$. There are $n m+n+m+1, n+m$-th degree Bernstein polynomials (see $[10,13]$ for details).

Some researchers have used the Bernstein polynomials of two variables in approximation theory (See [12,13]). But no result was known anything about the generating function of these polynomials. Note that for $k, j, n, m \in \mathrm{N}_{0}$, we have

$$
\begin{aligned}
& \frac{(t x)^{k}(t y)^{j} e^{2 t}}{k ! j ! e^{t(x+y)}} \\
= & \frac{t^{k} x^{k} t^{j} y^{j}}{k ! j !} e^{t(1-x)} e^{t(1-y)} \\
= & \frac{x^{k}}{k !}\left(t^{k} \sum_{n=0}^{\infty} \frac{(1-x)^{n}}{n !} t^{n}\right) \frac{y^{j}}{j !}\left(t^{j} \sum_{m=0}^{\infty} \frac{(1-y)^{m}}{m !} t^{m}\right) \\
= & \sum_{n=k m=j}^{\infty} \sum_{k, j ; n, m}^{\infty}(x, y) \frac{t^{n}}{n !} \frac{t^{m}}{m !}
\end{aligned}
$$

From the above, we obtain the generating function for $B_{k, j ; n, m}(x, y)$ as follows:

$$
\begin{aligned}
& F_{k, j}(t ; x, y)=\frac{(t x)^{k}(t y)^{j} e^{t(2-(x+y))}}{k ! j !} \\
= & \sum_{n=k}^{\infty} \sum_{m=j}^{\infty} B_{k, j ; n, m}(x, y) \frac{t^{n}}{n !} \frac{t^{m}}{m !}
\end{aligned}
$$

where $k, j, n, m \in \mathrm{N}_{0}$. We notice that,

$$
\begin{aligned}
& B_{k, j ; n, m}(x, y) \\
& =\left\{\begin{array}{cc}
\left(\begin{array}{l} 
\\
k
\end{array}\right)\left(\begin{array}{c}
m \\
j
\end{array}\right) x^{k} y^{j}(1-x)^{n-k}(1-y)^{m-j} & \text {,if } n \geq k \text { and } m \geq j \\
0 & \text { if } n<k \text { or } m<j
\end{array}\right.
\end{aligned}
$$

for $n, k, m, j \in \mathrm{N}_{0}$ (for details, see [9]).

Let $q \in(0,1)$. Then, $q$-integer of $x$ by $[x]_{q}:=\frac{1-q^{x}}{1-q}$ and $[x]_{-q}:=\frac{1-(-q)^{x}}{1+q}($ See $[2-18]$ for details $)$. Note that $\lim _{q \rightarrow 1}[x]_{q}=x$. [2] motivated us to write this paper and we have extended the results given in that paper to modified $q$-Bernstein polynomials of two variables.

\section{The Modified $q$-Bernstein Polynomials for Functions of Two Variables}

For $0 \leq k \leq n$ and $0 \leq j \leq m$, the $q$-Bernstein polynomials of degree $n+m$ are defined by

$$
\begin{aligned}
& B_{k, j ; n, m}(x, y ; q) \\
&=\left\{\begin{array}{c}
\left(\begin{array}{c}
n \\
k
\end{array}\right)\left(\begin{array}{c}
m \\
j
\end{array}\right)[x]_{q}^{k}[y]_{q}^{j}[1-x]_{q}^{n-k}[1-y]_{q}^{m-j}, \\
\text { if } n \geq k \text { and } m \geq j \\
0 \\
\text { if } n<k \text { or } m<j
\end{array}\right.
\end{aligned}
$$

For $q \in(0,1)$, consider the $q$-extension of (1.6) as follows:

$$
\begin{aligned}
& F_{k, j}(t, q ; x, y) \\
= & \frac{\left(t[x]_{q}\right)^{k}\left(t[y]_{q}\right)^{j}}{k ! j !} e^{t\left([1-x]_{q}+[1-y]_{q}\right)} \\
= & \frac{[x]_{q}^{k}}{k !}\left(\sum_{n=0}^{\infty} \frac{[1-x]_{q}^{n}}{n !} t^{n+k}\right) \frac{[y]_{q}^{j}}{j !}\left(\sum_{m=0}^{\infty} \frac{[1-y]_{q}^{m}}{m !} t^{m+j}\right) \quad(2.2) \\
= & \sum_{n=k m=j}^{\infty} \sum^{\infty}\left(\begin{array}{c}
n \\
k
\end{array}\right)\left(\begin{array}{c}
m \\
j
\end{array}\right)[x]_{q}^{k}[y]_{q}^{j}[1-x]_{q}^{n-k}[1-y]_{q}^{m-j} \frac{t^{n}}{n !} \frac{t^{m}}{m !} \\
\text { where } & k, j, n, m \in \mathrm{N}_{0} \\
& \lim _{q \rightarrow 1} F_{k, j}(t, q: x, y)=F_{k, j}(t ; x, y) .
\end{aligned}
$$

Definition 1. The modified $q$-Bernstein polynomials for functions of two variables is defined by means of the following generating function:

$$
\begin{aligned}
& F_{k, j}(t, q ; x, y) \\
= & \frac{\left(t[x]_{q}\right)^{k}\left(t[y]_{q}\right)^{j}}{k ! j !} e^{t\left([1-x]_{q}+[1-y]_{q}\right)} \\
= & \sum_{n=0}^{\infty} \sum_{m=0}^{\infty} B_{k, j ; n, m}(x, y ; q) \frac{t^{n}}{n !} \frac{t^{m}}{m !}
\end{aligned}
$$

where $k, j, n, m \in \mathrm{N}_{0}$.

By comparing the coefficients of (2.2) and (2.3), we obtain a formula for modified $q$-Bernstein polynomials of two variables given in the following theorem:

Theorem 1. For $k, j, n, m \in \mathrm{N}_{0}$, then, we have 


$$
\begin{aligned}
& B_{k, j ; n, m}(x, y ; q) \\
&\left\{\begin{array}{c}
\left(\begin{array}{l}
n \\
k
\end{array}\right)\left(\begin{array}{c}
m \\
j
\end{array}\right)[x]_{q}^{k}[y]_{q}^{j}[1-x]_{q}^{n-k}[1-y]_{q}^{m-j}, \\
\text { if } n \geq k \text { and } m \geq j \\
0 \\
\text { if } n<k \text { or } m<j
\end{array}\right.
\end{aligned}
$$

Theorem 2. (Recurrence Formula for $B_{k, j ; n, m}(x, y ; q)$ ) For $k, j, n, m \in \mathrm{N}_{0}$, we have

$$
\begin{aligned}
& B_{k, j ; n, m}(x, y ; q) \\
= & {[1-x]_{q}[1-y]_{q} B_{k, j ; n-1, m-1}(x, y ; q) } \\
& +[1-x]_{q}[y]_{q} B_{k, j-1 ; n-1, m-1}(x, y ; q) \\
& +[x]_{q}[1-y]_{q} B_{k-1, j ; n-1, m-1}(x, y ; q) \\
& +[x]_{q}[y]_{q} B_{k-1, j-1 ; n-1, m-1}(x, y ; q) .
\end{aligned}
$$

Proof. By using the definition of Bernstein polynomials for functions of two variables defined by (2.4), we have

$$
\begin{aligned}
& B_{k, j ; n, m}(x, y ; q) \\
= & \left(\begin{array}{c}
n \\
k
\end{array}\right)\left(\begin{array}{c}
m \\
j
\end{array}\right)[x]_{q}^{k}[y]_{q}^{j}[1-x]_{q}^{n-k}[1-y]_{q}^{m-j} \\
= & {\left[\left(\begin{array}{c}
n-1 \\
k
\end{array}\right)+\left(\begin{array}{c}
n-1 \\
k-1
\end{array}\right)\right]\left[\left(\begin{array}{c}
m-1 \\
j
\end{array}\right)+\left(\begin{array}{c}
m-1 \\
j-1
\end{array}\right)\right] } \\
& {[x]_{q}^{k}[y]_{q}^{j}[1-x]_{q}^{n-k}[1-y]_{q}^{m-j} } \\
= & {[1-x]_{q}[1-y]_{q} B_{k, j ; n-1, m-1}(x, y ; q) } \\
& +[1-x]_{q}[y]_{q} B_{k, j-1 ; n-1, m-1}(x, y ; q) \\
& +[x]_{q}[1-y]_{q} B_{k-1, j ; n-1, m-1}(x, y ; q) \\
& +[x]_{q}[y]_{q} B_{k-1, j-1 ; n-1, m-1}(x, y ; q) .
\end{aligned}
$$

Theorem 3. For $k, j, n, m \in \mathrm{N}_{0}$, we get

$$
B_{n-k, m-j ; n, m}(1-x, 1-y ; q)=B_{k, j ; n, m}(x, y ; q)
$$

and

$$
\begin{aligned}
& \mathbf{B}_{n, m}(1: x, y, q) \\
= & \left(1+(1-q)[x]_{q}[1-x]_{q}\right)^{n} \\
& \times\left(1+(1-q)[y]_{q}[1-y]_{q}\right)^{m} .
\end{aligned}
$$

Proof. Let $f$ be a continuous function of two variables on $D \times D$. Then the modified $q$-Bernstein operator of order $n+m$ for $f$ is defined by

$$
\begin{aligned}
& \mathbf{B}_{n, m}(f: x, y, q) \\
= & \sum_{k=0}^{n} \sum_{j=0}^{m} f\left(\frac{k}{n}, \frac{j}{m}\right) B_{k, j ; n, m}(x, y ; q)
\end{aligned}
$$

where $0 \leq x \leq 1, \quad 0 \leq y \leq 1, n, m \in \mathrm{N}$. From Theorem 1 and the definition of modified $q$-Bernstein operator given by (2.6) for $f(x, y)=x y$, we have

$$
\begin{aligned}
& \mathbf{B}_{n, m}(f: x, y, q) \\
= & \sum_{k=0}^{n} \sum_{j=0}^{m} f\left(\frac{k}{n}, \frac{j}{m}\right)\left(\begin{array}{c}
n \\
k
\end{array}\right)\left(\begin{array}{c}
m \\
j
\end{array}\right) \\
& {[x]_{q}^{k}[1-x]_{q}^{n-k}[y]_{q}^{k}[1-y]_{q}^{m-j} } \\
= & {[x]_{q}\left(1-[1-x]_{q}[x]_{q}(q-1)\right)^{n-1} } \\
& \times[y]_{q}\left(1-[1-y]_{q}[y]_{q}(q-1)\right)^{m-1} \\
= & f\left([x]_{q},[y]_{q}\right)\left(1+(1-q)[x]_{q}[1-x]_{q}\right)^{n-1} \\
& \times\left(1+(1-q)[y]_{q}[1-y]_{q}\right)^{m-1}
\end{aligned}
$$

From Theorem 1, we have

$$
\begin{aligned}
\mathbf{B}_{n, m}(1: x, y, q)= & \sum_{k=0}^{n} \sum_{j=0}^{m} B_{k, j ; n, m}(x, y ; q) \\
= & \sum_{k=0}^{n} B_{k, n}(x, q) \sum_{j=0}^{m} B_{j, m}(y, q) \\
= & \left(1+(1-q)[x]_{q}[1-x]_{q}\right)^{n} \\
& \left(1+(1-q)[y]_{q}[1-y]_{q}\right)^{m} .
\end{aligned}
$$

The modified $q$-Bernstein polynomials of two variables are symmetric polynomials:

$$
\begin{aligned}
& B_{n-k, m-j ; n, m}(1-x, 1-y ; q) \\
= & \left(\begin{array}{c}
n \\
n-k
\end{array}\right)[x]_{q}^{k}[1-x]_{q}^{n-k}\left(\begin{array}{c}
n \\
m-j
\end{array}\right)[y]_{q}^{j}[1-y]_{q}^{m-j} \\
= & \left(\begin{array}{c}
n \\
k
\end{array}\right)[1-x]_{q}^{k}[x]_{q}^{n-k}\left(\begin{array}{c}
n \\
j
\end{array}\right)[1-y]_{q}^{j}[y]_{q}^{m-j} \\
= & B_{k, j ; n, m}(x, y ; q) .
\end{aligned}
$$

by replacing $k$ by $n-k$ and $j$ by $m-j$.

Theorem 4. For $\xi, \rho \in \mathrm{C}$, and for $n, m \in \mathrm{N}$, then, we procure

$$
\begin{aligned}
& B_{k, j ; n, m}(x, y ; q) \\
= & -\frac{n ! m !}{4 \pi^{2}} \oint_{C C} \frac{\left([x]_{q} \xi\right)^{k}\left([y]_{q} \rho\right)^{j}}{k ! j !} e^{\left([1-x]_{q} \xi+[1-y]_{q} \rho\right)} \frac{d \xi}{\xi^{n+1}} \frac{d \rho}{\rho^{m+1}}
\end{aligned}
$$

where $C$ is a circle around the origin and integration is in the positive direction.

Proof. By using the definition of the modified $q$-Bernstein polynomials of two variables and the basic theory of complex analysis including Laurent series that

$$
\begin{aligned}
& \oint_{C} \oint_{C} \frac{\left([x]_{q} \xi\right)^{k}\left([y]_{q} \rho\right)^{j}}{k ! j !} e^{\left([1-x]_{q} \xi+[1-y]_{q} \rho\right)} \frac{d \xi}{\xi^{n+1}} \frac{d \rho}{\rho^{m+1}} \\
= & \sum_{l=0}^{\infty} \sum_{r=0}^{\infty} \oint_{C} \oint_{C} \frac{B_{k, l}(x, q) \xi^{l}}{l !} \frac{B_{j, r}(y, q) \rho^{r}}{r !} \frac{d \xi}{\xi^{n+1}} \frac{d \rho}{\rho^{m+1}} \\
= & (2 \pi i)^{2}\left(\frac{B_{k, j ; n, m}(x, y ; q)}{n ! m !}\right) .
\end{aligned}
$$


By using (2.7) and (2.8), we obtain

$$
\begin{aligned}
& \frac{n ! m !}{(2 \pi i)^{2}} \oint_{C} \oint_{C} \frac{\left([x]_{q} \xi\right)^{k}}{k !} \frac{\left([y]_{q} \rho\right)^{j}}{j !} \frac{d \xi}{\xi^{n+1}} \frac{d \rho}{\rho^{m+1}} \\
= & B_{k, j ; n m}(x, y ; q)
\end{aligned}
$$

and

$$
\begin{aligned}
& \oint_{C} \oint_{C} \frac{\left([x]_{q} \xi\right)^{k}}{k !} \frac{\left([y]_{q} \rho\right)^{j}}{j !} e^{\left([1-x]_{q} \xi+[1-y]_{q} \rho\right)} \frac{d \xi}{\xi^{n+1}} \frac{d \rho}{\rho^{m+1}} \\
& =(2 \pi i)^{2}\left(\frac{[x]_{q}^{k}[y]_{q}^{j}[1-x]_{q}^{n-k}[1-y]_{q}^{m-j}}{k ! j !(n-k) !(m-j) !}\right) .
\end{aligned}
$$

We also obtain from (2.5) and (2.9) that

$$
\begin{aligned}
& \frac{n ! m !}{(2 \pi i)^{2}} \oint_{C} \oint_{C} \frac{\left([x]_{q} \xi\right)^{k}\left([y]_{q} \rho\right)^{j}}{k ! j !} e^{\left([1-x]_{q} \xi+[1-y]_{q} \rho\right)} \frac{d \xi}{\xi^{n+1}} \frac{d \rho}{\rho^{m+1}} \\
= & \left(\begin{array}{c}
n \\
k
\end{array}\right)\left(\begin{array}{c}
m \\
j
\end{array}\right)[x]_{q}^{k}[1-x]_{q}^{n-k}[y]_{q}^{j}[1-y]_{q}^{m-j} .
\end{aligned}
$$

Therefore we see that from (2.8) and (2.10) that

$$
\begin{aligned}
& B_{k, j ; n, m}(x, y ; q) \\
= & \left(\begin{array}{c}
n \\
k
\end{array}\right)\left(\begin{array}{c}
m \\
j
\end{array}\right)[x]_{q}^{k}[1-x]_{q}^{n-k}[y]_{q}^{j}[1-y]_{q}^{m-j} .
\end{aligned}
$$

Theorem 5. (The Derivative Formula for $B_{k, j ; n, m}(x, y ; q)$ ) For $k, j, n, m \in \mathrm{N}$, then, we derive the following

$$
\begin{aligned}
& \frac{\partial^{2}}{\partial x \partial y}\left(B_{k, j ; n, m}(x, y ; q)\right) \\
= & n m\left(q^{x+y} B_{k-1, j-1 ; n-1, m-1}(x, y ; q)\right. \\
& -q^{x-y+1} B_{k-1, j ; n-1, m-1}(x, y ; q) \\
& -q^{1-x+y} B_{k, j-1 ; n-1, m-1}(x, y ; q) \\
& \left.+q^{2-(x+y)} B_{k, j ; n-1, m-1}(x, y ; q)\right) \frac{\ln ^{2} q}{(q-1)^{2}} .
\end{aligned}
$$

Proof. Using the definition of modified $q$-Bernstein polynomials for functions of two variables and the property (1.3), we have

$$
\begin{aligned}
& \frac{\partial^{2}}{\partial x \partial y}\left(B_{k, j ; n, m}(x, y ; q)\right) \\
= & \frac{\partial^{2}}{\partial x \partial y}\left(B_{k, n}(x ; q) B_{j, m}(y ; q)\right) \\
= & \frac{d}{d x}\left(B_{k, n}(x ; q)\right) \frac{d}{d y}\left(B_{j, m}(y ; q)\right)
\end{aligned}
$$

and after some calculations, the proof is complete.

Therefore, we can write the modified $q$-Bernstein polynomials for functions of two variables as a linear combination of polynomials of higher order as follows:

Theorem 6. For $k, j, n, m \in \mathrm{N}_{0}$, we have

$$
\begin{aligned}
& \left(1+(1-q)[x]_{q}[1-x]_{q}\right) \\
& \left(1+(1-q)[y]_{q}[1-y]_{q}\right) B_{k, j ; n, m}(x, y ; q) \\
= & \left(\frac{n-k+1}{n+1}\right)\left(\frac{m-j+1}{m+1}\right) B_{k, j ; n+1, m+1}(x, y ; q) \\
+ & \left(\frac{n-k+1}{n+1}\right)\left(\frac{j+1}{m+1}\right) B_{k, j+1 ; n+1, m+1}(x, y ; q) \\
& +\left(\frac{k+1}{n+1}\right)\left(\frac{m-j+1}{m+1}\right) B_{k+1, j ; n+1, m+1}(x, y ; q) \\
& +\left(\frac{k+1}{n+1}\right)\left(\frac{j+1}{m+1}\right) B_{k+1, j+1 ; n+1, m+1}(x, y ; q) .
\end{aligned}
$$

Proof. It follows after expanding the series and some algebraic operations.

Theorem 7. For $k, j, n, m \in \mathrm{N}_{0}$, we have

$$
\begin{aligned}
& B_{k, j ; n, m}(x, y ; q) \\
= & \left(\frac{n-k+1}{k}\right)\left(\frac{m-j+1}{j}\right)\left(\frac{[x]_{q}[y]_{q}}{[1-x]_{q}[1-y]_{q}}\right) B_{k-1, j-1 ; n, m}(x, y ; q) .
\end{aligned}
$$

Proof. To prove this theorem, we start with the right hand side:

$$
\begin{aligned}
& \left(\frac{n-k+1}{k}\right)\left(\frac{m-j+1}{j}\right)\left(\frac{[x]_{q}[y]_{q}}{[1-x]_{q}[1-y]_{q}}\right) \\
& B_{k-1, j-1 ; n, m}(x, y ; q) \\
= & \frac{n !}{(n-k) ! k !} \cdot \frac{m !}{(m-j) ! j !}\left(\frac{[x]_{q}[y]_{q}}{[1-x]_{q}[1-y]_{q}}\right) \\
& {\left[\begin{array}{c}
x]_{q}^{k-1}[y]_{q}^{j-1}[1-x]_{q}^{n-k+1}[1-y]_{q}^{m-j+1} \\
=
\end{array}\right.} \\
& \left(\begin{array}{c}
n \\
k
\end{array}\right)\left(\begin{array}{c}
m \\
j
\end{array}\right)[x]_{q}^{k}[y]_{q}^{j}[1-x]_{q}^{n-k}[1-y]_{q}^{m-j}=B_{k, j ; n, m}(x, y ; q) .
\end{aligned}
$$

Theorem 8. For $k, j, n, m \in \mathrm{N}_{0}$, we obtain

$$
\begin{aligned}
& B_{k, j ; n, m}(x, y ; q) \\
& =\sum_{l=k r=j}^{n} \sum_{q^{(l-k)(1-x)+(r-j)(1-y)}\left[\begin{array}{l}
n \\
l
\end{array}\right)\left(\begin{array}{l}
l \\
k
\end{array}\right)\left(\begin{array}{c}
m \\
j
\end{array}\right)\left(\begin{array}{l}
j \\
r
\end{array}\right)(-1)_{q}^{l-k+r-j}[y]_{q}^{r}} .
\end{aligned}
$$

Proof. From the definition of modified $q$-Bernstein polynomials of two variables and binomial theorem with $k, j, n, m \in \mathrm{N}_{0}$, we have

$$
\begin{aligned}
& B_{k, j ; n, m}(x, y ; q) \\
= & \left(\begin{array}{c}
n \\
k
\end{array}\right)\left(\begin{array}{c}
m \\
j
\end{array}\right)[x]_{q}^{k}[1-x]_{q}^{n-k}[y]_{q}^{j}[1-y]_{q}^{m-j} \\
= & \left(\begin{array}{c}
n \\
k
\end{array}\right)\left(\begin{array}{c}
m \\
j
\end{array}\right)[x]_{q}^{k}[y]_{q}^{j}\left(1-q^{1-x}[x]\right)^{n-k}\left(1-q^{1-y}[y]\right)^{m-j} \\
= & \sum_{l=k}^{n} \sum_{r=j}^{m}\left(\begin{array}{l}
n \\
l
\end{array}\right)\left(\begin{array}{l}
l \\
k
\end{array}\right)\left(\begin{array}{c}
m \\
j
\end{array}\right)\left(\begin{array}{l}
j \\
r
\end{array}\right)(-1)^{l-k+r-j}(1-k)(1-x)+(r-j)(1-y)_{[x]_{q}^{l}[y]_{q}^{r}}
\end{aligned}
$$

Theorem 9. The following identity 


$$
\begin{aligned}
& \left([x]_{q}[y]_{q}\right)^{l}=\frac{1}{\left([1-x]_{q}+[x]_{q}\right)^{n-l}\left([1-y]_{q}+[y]_{q}\right)^{m-l}} \\
& \sum_{k=l}^{n} \sum_{j=l}^{m} \frac{\left(\begin{array}{l}
k \\
l
\end{array}\right)\left(\begin{array}{l}
j \\
l
\end{array}\right)}{\left(\begin{array}{l}
m \\
l
\end{array}\right)} B_{k, j ; n, m}(x, y ; q)
\end{aligned}
$$

is true.

Proof. We easily see that from the property of the modified $q$-Bernstein polynomials of two variables that

$$
\begin{aligned}
& \sum_{k=1}^{n} \sum_{j=1}^{m} \frac{k j}{n m} B_{k, j ; n, m}(x, y ; q) \\
= & \sum_{k=1}^{n} \sum_{j=1}^{m}\left(\begin{array}{c}
n-1 \\
k-1
\end{array}\right)\left(\begin{array}{c}
m-1 \\
j-1
\end{array}\right)[x]_{q}^{k}[y]_{q}^{j}[1-x]_{q}^{n-k}[1-y]_{q}^{m-j} \\
= & {[x]_{q}[y]_{q}\left([x]_{q}+[1-x]_{q}\right)^{n-1}\left([y]_{q}+[1-y]_{q}\right)^{m-1} }
\end{aligned}
$$

and that

$$
\begin{aligned}
& \sum_{k=2}^{n} \sum_{j=2}^{m} \frac{\left(\begin{array}{l}
k \\
2
\end{array}\right)\left(\begin{array}{l}
j \\
2 \\
2
\end{array}\right)\left(\begin{array}{l}
m \\
2
\end{array}\right)}{(k, j ; n, m}(x, y ; q) \\
= & \sum_{k=2}^{n} \sum_{j=2}^{m}\left(\begin{array}{l}
n-2 \\
k-2
\end{array}\right)\left(\begin{array}{c}
m-2 \\
j-2
\end{array}\right)[x]_{q}^{k}[y]_{q}^{j}[1-x]_{q}^{n-k}[1-y]_{q}^{m-j} \\
= & {[x]_{q}^{2}[y]_{q}^{2}\left([x]_{q}+[1-x]_{q}\right)^{n-2}\left([y]_{q}+[1-y]_{q}\right)^{m-2} }
\end{aligned}
$$

Continuing this way, we have

$$
\begin{aligned}
& \sum_{k=l}^{n} \sum_{j=l}^{m} \frac{\left(\begin{array}{l}
k \\
l
\end{array}\right)\left(\begin{array}{l}
j \\
l
\end{array}\right)}{\left(\begin{array}{l}
n \\
l
\end{array}\right)\left(\begin{array}{l}
m \\
l
\end{array}\right)} B_{k, j ; n, m}(x, y ; q) \\
= & {[x]_{q}^{l}[y]_{q}^{l}\left([x]_{q}+[1-x]_{q}\right)^{n-l}\left([y]_{q}+[1-y]_{q}\right)^{m-l} }
\end{aligned}
$$

and after some algebraic operations, we obtain the desired result.

We see that from the theorem above, it is possible to write $\left([x]_{q}[y]_{q}\right)^{k}$ as a linear combination of the two variables modified $q$-Bernstein polynomials.

For $k \in \mathrm{N}_{0}$, the Bernoulli polynomials of degree $k$ are defined by

$$
\begin{aligned}
& \left(\frac{t}{e^{t}-1}\right)^{k} e^{x t} \\
= & \underbrace{\left(\frac{t}{e^{t}-1}\right) \times \cdots \times\left(\frac{t}{e^{t}-1}\right)}_{k-\text { times }} e^{x t} \\
= & \sum_{n=0}^{\infty} B_{n}^{(k)}(x) \frac{t^{n}}{n !},
\end{aligned}
$$

and $B_{n}^{(k)}=B_{n}^{(k)}(0)$ are called the n-th Bernoulli numbers of order $k$. It is well known that the second kind Stirling numbers are defined by

$$
\frac{\left(e^{t}-1\right)^{k}}{k !}:=\sum_{n=0}^{\infty} S(n, k) \frac{t^{n}}{n !}(2.11)
$$

for $k \in \mathrm{N}$ (see [2]). By using the above relations we can give the following theorem:

Theorem 10. For $k, j, n, m \in \mathrm{N}_{0}$, we have

$$
\begin{aligned}
& B_{k, j ; n, m}(x, y ; q) \\
= & {[x]_{q}^{k}[y]_{q}^{j} \sum_{l=0}^{n} \sum_{r=0}^{m}\left(\begin{array}{l}
n \\
l
\end{array}\right)\left(\begin{array}{c}
m \\
r
\end{array}\right) } \\
& \times B_{l}^{(k)}\left([1-x]_{q}\right) B_{r}^{(j)}\left([1-y]_{q}\right) S(n-l, k) S(m-r, j) .
\end{aligned}
$$

Proof. By using the generating function of modified $q$ Bernstein polynomials of two variables, we have

$$
\begin{aligned}
& \frac{\left(t[x]_{q}\right)^{k}\left(t[y]_{q}\right)^{j}}{k ! j !} e^{t\left([1-x]_{q}+[1-y]_{q}\right)} \\
= & {[x]_{q}^{k}[y]_{q}^{j}\left(\sum_{n=0}^{\infty} S(n, k) \frac{t^{n}}{n !}\right)\left(\sum_{m=0}^{\infty} S(m, j) \frac{t^{m}}{m !}\right) } \\
& \times\left(\sum_{l=0}^{\infty} B_{l}^{(k)}\left([1-x]_{q}\right) \frac{t^{l}}{l !}\right)\left(\sum_{r=0}^{\infty} B_{r}^{(j)}\left([1-y]_{q}\right) \frac{t^{r}}{r !}\right) \\
= & \sum_{n \geq k m \geq j} \sum_{k, j ; n, m} B_{m}(x, y ; q) \frac{t^{n}}{n !} \frac{t^{m}}{m !}
\end{aligned}
$$

by using the Cauchy product. By comparing last two relations, we have the desired result.

Let $\Delta$ be the shift difference operator defined by $\Delta f(x)=f(x+1)-f(x)$. By using the iterative method we have

$$
\Delta^{n} f(0)=\sum_{k=0}^{n}\left(\begin{array}{l}
n \\
k
\end{array}\right)(-1)^{n-k} f(k),
$$

for $n \in \mathrm{N}$.

$$
\begin{aligned}
& \sum_{n=0}^{\infty} S(n, k) \frac{t^{n}}{n !}=\frac{1}{k !} \sum_{l=0}^{k}\left(\begin{array}{l}
k \\
l
\end{array}\right)(-1)^{k-l} e^{l t} \\
= & \sum_{n=0}^{\infty}\left\{\frac{1}{k !} \sum_{l=0}^{k}\left(\begin{array}{l}
k \\
l
\end{array}\right)(-1)^{k-l} l^{n}\right\} \frac{t^{n}}{n !}=\sum_{n=0}^{\infty} \frac{\Delta^{k} 0^{n}}{k !} \frac{t^{n}}{n !} .
\end{aligned}
$$

By comparing the coefficients on both sides above, we have

$$
S(n, k)=\frac{\Delta^{k} 0^{n}}{k !}
$$

for $n, k \in \mathrm{N}_{0}$. By using the equations (2.11) and (2.12), we obtain the following relation

$$
\begin{aligned}
& B_{k, j ; n, m}(x, y ; q) \\
= & {[x]_{q}^{k}[y]_{q}^{j} \sum_{l=0}^{n} \sum_{r=0}^{m}\left(\begin{array}{l}
n \\
l
\end{array}\right)\left(\begin{array}{c}
m \\
r
\end{array}\right) } \\
& \times B_{l}^{(k)}\left([1-x]_{q}\right) B_{r}^{(j)}\left([1-y]_{q}\right) \frac{\Delta^{k} 0^{n-l}}{k !} \frac{\Delta^{j} 0^{m-r}}{j !}
\end{aligned}
$$


which is the relation of the $q$-Bernstein polynomials of two variables in terms of Bernoulli polynomials of order $k$ and second Stirling numbers with shift difference operator.

Let $(E h)(x)=h(x+1)$ be the shift operator. Then the $q$-difference operator is defined by

$$
\Delta_{q}^{n}=\prod_{j=0}^{n-1}\left(E-q^{j} I\right)
$$

where $I$ is and identity operator ( See [2]).

For $f \in C[0,1]$ and $n \in \mathrm{N}$, we have

$$
\Delta_{q}^{n} f(0)=\sum_{k=0}^{n}\left(\begin{array}{l}
n \\
k
\end{array}\right)_{q}(-1)^{k} q^{\left(\begin{array}{l}
n \\
2
\end{array}\right)} f(n-k)
$$

where $\left(\begin{array}{l}n \\ k\end{array}\right)_{q}$ is called the Gaussian binomial coefficients, which are defined by

$$
\left(\begin{array}{l}
n \\
k
\end{array}\right)_{q}=\frac{[x]_{q}[x-1]_{q} \cdots[x-k+1]_{q}}{[k]_{q} !} .
$$

Theorem 11. For $n, m, l, r \in \mathrm{N}_{0}$, we have

$$
\begin{aligned}
& \frac{1}{\left([x]_{q}+[1-x]_{q}\right)^{n-l}\left([y]_{q}+[1-y]_{q}\right)^{m-l}} \\
& \sum_{k=l}^{n} \sum_{j=l}^{m} \frac{\left(\begin{array}{l}
k \\
l
\end{array}\right)\left(\begin{array}{l}
j \\
l
\end{array}\right)}{\left(\begin{array}{l}
m \\
l
\end{array}\right)} B_{k, j ; n, m}(x, y ; q)
\end{aligned}
$$

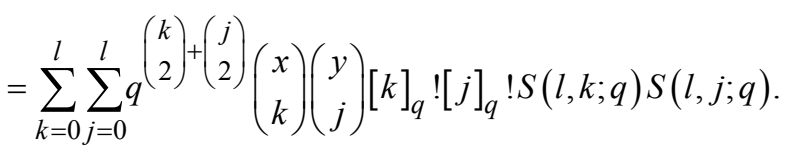

Proof. Let $F_{q}(t)$ be the generating function of the $q$ extension of the second kind Stirling numbers as follows:

$$
\begin{aligned}
F_{q}(t) & :=\frac{q^{-\left(\begin{array}{l}
k \\
2
\end{array}\right)}}{[k]_{q} !} \sum_{j=0}^{k}(-1)^{k-j}\left(\begin{array}{c}
k \\
j
\end{array}\right)_{q} q^{\left(\begin{array}{c}
k-j \\
2
\end{array}\right)} e^{[i]_{q} t} \\
& =\sum_{n=0}^{\infty} S(n, k ; q) \frac{t^{n}}{n !}
\end{aligned}
$$

From the above, we have

$$
S(n, k ; q)=\frac{q^{-\left(\begin{array}{l}
k \\
2
\end{array}\right)}}{[k]_{q} !} \sum_{j=0}^{k}(-1)^{j} q^{\left(\begin{array}{l}
j \\
2
\end{array}\right)}\left(\begin{array}{l}
k \\
j
\end{array}\right)_{q}[k-j]_{q}^{n}=\frac{q^{\left(\begin{array}{l}
k \\
2
\end{array}\right)}}{[k]_{q} !} \Delta_{q}^{k} 0^{n}
$$

where $[k]_{q} !=[k]_{q}[k-1]_{q} \cdots[2]_{q}[1]_{q}$. It is easy to see that

$$
[x]_{q}^{n}=\sum_{k=0}^{n} q^{\left(\begin{array}{l}
k \\
2
\end{array}\right)}\left(\begin{array}{l}
x \\
k
\end{array}\right)_{q}[k]_{q} ! S(n, k ; q)
$$

by similar way

$$
[y]_{q}^{j}=\sum_{r=0}^{j} q^{\left(\begin{array}{l}
r \\
2
\end{array}\right)}\left(\begin{array}{l}
y \\
r
\end{array}\right)_{q}[r]_{q} ! S(j, r ; q) .
$$

We have above equality. Then, we obtain the desired result in Theorem from the equations (2.18), (2.19) and Theorem 7.

\section{Interpolation Function of Modified q- Bernstein Polynomial for Functions of Two Variables}

For $s \in \mathrm{C}$, and $x \neq 1, y \neq 1$, by applying the Mellin transformation to generating function of Bernstein polynomials of two variables, we get

$$
\begin{aligned}
S_{q}(s, k, j ; x, y) & =\frac{1}{\Gamma(s)} \int_{0}^{\infty} F_{k, j}(-t, q ; x, y) t^{s-k-j-1} d t \\
& =\frac{(-1)^{k+j}[x]_{q}^{k}[y]_{q}^{j}}{k ! j !}\left([1-x]_{q}+[1-y]_{q}\right)^{-s} .
\end{aligned}
$$

By using the equation (3.1), we define the interpolation function of the polynomials $B_{k, j ; n, m}(x, y ; q)$ as follows:

Definition 2. Let $s \in \mathrm{C}$ and $x \neq 1, y \neq 1$, we define

$$
S_{q}(s, k, j ; x, y)=\frac{[x]_{q}^{k}[y]_{q}^{j}}{k ! j !}(-1)^{k+j}\left([1-x]_{q}+[1-y]_{q}\right)^{-s} \text {. }
$$

By using (3.2), we have $S_{q}(s, k, j ; x, y) \rightarrow S(s, k, j ; x, y)$ as $q \rightarrow 1$. Thus one has

$$
S(s, k, j ; x, y)=\frac{(-1)^{k+j}}{k ! j !} x^{k} y^{j}(2-(x+y))^{-s} .
$$

By substituting $x=1$ and $y=1$ into the above, we have $S(s, k, j ; x, y)=\infty$.

We now evaluate the $m$ th s-derivatives of $S(s, k, j ; x, y)$ as follows:

$$
\frac{\partial^{m}}{\partial s^{m}} S(s, k, j ; x, y)=\log ^{m}\left(\frac{1}{2-(x+y)}\right) S(s, k, j ; x, y)
$$

where $x \neq 1$ and $y \neq 1$.

\section{References}

[1] Kim, T., On the q-extension of Higher-Order Euler polynomials, Proceedings of the Jangjeon Mathematical Society, 15 (2012), no. 3, pp. 293-302.

[2] Kim, T., Jang, L.-C., and Yi, H., Note on the modified q-Bernstein polynomials, Discrete Dyanmics in Nature and Society, Volume 2010 (2010), Article ID 706483, 12 pages.

[3] Kim, T., A note q-Bernstein polynomials, Russ. J. Math. Phys. 18(2011), pp. 41-50.

[4] Kim, T., Choi, J. and Kim, Y. H., Some identities on the qBernstein polynomials, q-Stirling numbers and q-Bernoulli numbers, Adv. Stud. Contemp. Math. 20(2010), pp. 335-341. 
[5] Kim, T., Choi, J. and Kim, Y. H., q-Bernstein Polynomials Associated with q-Stirling Numbers and Carlitz's q-Bernoulli Numbers, Abstract and Applied Analysis, Article ID 150975, 11 pages.

[6] Kim, T., Choi, J., Kim, Y. H. and Ryoo, C. S., On the fermionic padic integral representation of Bernstein polynomials associated with Euler numbers and polynomials, J. Inequal. Appl. 2010 (2010), Art ID 864247, 12 pages.

[7] Kim, T., Some identities on the q-Euler polynomials of higher order and q-Stirling numbers by the fermionic p-adic integral on $Z_{\mathrm{p}}$, Russ. J. Math. Phys., 16 (2009), no.4, pp. 484-491.

[8] Acikgoz, M., and Araci, S., On the generating function of the Bernstein polynomials, Numerical Analysis and Applied Mathematics, International conference 2010, pp. 1141-1143.

[9] Acikgoz, M., and Araci, S., New generating function of Bernstein type polynomials for two variables, Numerical Analysis and Applied Mathematics, International conference 2010, pp. 11331136.

[10] Acikgoz, M., and Araci, S., A study on the integral of the product of several type Bernstein polynomials, IST Transaction of Applied Mathematics Modelling and Simulation,2010, vol. 1, no. 1(2), ISSN 1913-8342, pp. 10-14.

[11] Acikgoz, M., and Simsek, M., On multiple interpolation functions of the Nörlund-type q-Euler polynomials, Abstr. Appl. Anal. 2009, Art. ID 382574, 14 pages.
[12] Buyukyazici, İ., and İbikli, E., Bernstein polynomials of two variable functions, Graduate School of Natural and Applied Sciences, Department of Mathematics, 1999, 49 pages, Ankara, Turkey.

[13] Buyukyazici, İ., and İbikli, E., The approximation properties of generalized Bernstein polynomials of two variables, Applied Math. and Comput. 156 (2004) 367-380.

[14] Ryoo, C. S., A note on the weighted $q$-Euler numbers and polynomials, Adv. Stud. Contemp. Math. 21 (2011), pp. 47-54.

[15] Oruc, H., and Phillips, G. M., A generalization of the Bernstein polynomials, Proceedings of the Edinburgh Mathematical society (1999) 42, 403-413.

[16] Ostrovska, S., On the q-Bernstein polynomials, Adv. Stud. Contemp. Math. 11 (2) (2005), pp. 193-204.

[17] Phillips, G. M., A survey of results on the q-Bernstein polynomials, IMA Journal of Numerical Analysis Advance Access published online on June 23, (2009), pp. 1-12.

[18] Simsek, Y., and Acikgoz, M., A new generating function of qBernstein-type polynomials and their interpolation function, Abstract and Applied Analysis, volume 2010, Article ID 769095, 12 pages. 